\begin{tabular}{|l|l|}
\hline $\begin{array}{l}\text { Instituto de } \\
\text { Geriatria e Gerontologia }\end{array}$ & $\begin{array}{l}\text { Pan American Journal of Aging Research } \\
\text { PAJAR, Porto Alegre, v. 8, p. 1-9, jan.-dez. } 2020 \\
\text { ISSN-L: 2357-9641 }\end{array}$ \\
\hline del http://dx.doi.org/10.15448/2357-9641.2020.1.34163 & \\
\cline { 1 - 2 }
\end{tabular}

ARTIGO ORIGINAL

\title{
Conhecimento de estudantes de medicina sobre medicamentos inapropriados para idosos
}

\author{
Medical students' knowledge about inappropriate medicines for elderly \\ Conocimiento de estudiantes de medicina sobre medicamentos inapropiados para ancianos
}

Isadora Brandão da

Silva ${ }^{1}$

orcid.org/0000-0003-0237-6143

isabrandao.dasilva@gmail.com

\section{Geniffer Curtinaz \\ Cardoso ${ }^{1}$}

orcid.org/0000-0002-8258-8121 geniffermed@gmail.com

\section{Felipe Franco ${ }^{1}$}

orcid.org/0000-0001-7656-1662 francofelip@gmail.com

\section{Paulo Roberto Cardoso Consoni ${ }^{1}$}

orcid.org/0000-0002-6028-061X consoniconsoni@gmail.com

Recebido em: 29 mai. 2019. Aprovado em: 10 set. 2019. Publicado em: 03 agos. 2020.

\begin{abstract}
Conhecimento sobre Medicamentos Inapropriados para Idosos Resumo

Objetivos: verificar e descrever o conhecimento de alunos de medicina sobre medicamentos inapropriados para idosos conforme os Critérios de Beers.

Métodos: estudo transversal com a aplicação de questionários a 102 alunos matriculados do $9 .^{\circ}$ ao $11 .^{\circ}$ semestre do curso de Medicina da Universidade Luterana do Brasil - Canoas. Levantaram-se informações referentes ao conhecimento dos acadêmicos sobre medicamentos inapropriados para idosos frágeis ou com mais de 80 anos, com base na classificação do Critério de Beers.

Resultados: $84.3 \%$ dos alunos pesquisados afirmaram ter conhecimento sobre medicamentos inapropriados para idosos, porém o percentual total de acertos foi de $20,2 \%$. O maior número de acertos foi relacionado quanto à segurança do Diazepam (49\%) e o menor percentual de acertos foi quanto ao medicamento Fluoxetina, com apenas $4.9 \%$ das respostas corretas. O maior percentual de respostas "Não Sei" foram relacionados em Oxibutinina (48\%) e Ciclobenzaprina (37,3\%). Os entrevistados que afirmaram não conhecer o tema e os individuos do sexo masculino apresentaram conhecimento significativamente maior com p=0,001. Conclusões: a maioria dos entrevistados afirmaram possuir conhecimento sobre o tema, porém a frequência de acertos foi baixa. O aprimoramento do ensino visando maior qualidade na prescrição e o uso racional de medicamentos é necessário para minimizar a iatrogenia em idosos.
\end{abstract}

Palavras-chave: geriatria, idoso, iatrogenia, uso de medicamentos, farmacoterapia, educação médica. Abstract
Aims: Verify and describe the knowledge of medical students about inappropriate
medicines for the elderly according to the Beers Criteria.
Methods: Cross-sectional study with questionnaires applied to 102 students
enrolled from the gth to the 11 th semester of medical course of the Universida-
de Luterana do Brasil - Canoas. Sought information referring at the academic
knowledge about inappropriate medications for frail elderly or over 80 years of
age, based on the classification of the Beers Criteria.
Results: $84.3 \%$ of the students surveyed said they have knowledge about inappro-
priate medication for elderly, however the total percentage of questionnaires with
correct answers was $20.2 \%$. The greatest number of correct answers was related
to security level of Diazepam ( $49 \%$ ) and the lowest percentage of correct answers
was about Fluoxetine, with only $4,9 \%$ of correct answers. The higher percentage
of answers "I don't know" were Oxybutynin ( $48 \%$ ) and Cyclobenzaprine ( $37,3 \%$ ).
The interviewees who said they didn't know the theme and male individuals
showed significantly greater knowledge with p=0,001.
Conclusion: The majority of surveyed affirmed that they had knowledge about
the theme, but the frequency of hits was low. The improvement of medical edu-
cation, aiming increased quality in prescribing and rational use of medicines, is
necessary to minimize the iatrogenic in elderly patients.
Keywords: geriatrics, aged, iatrogenic disease, drug utilization, drug therapy education, medical.
Artigo está licenciado sob forma de uma licença Creative Commons Atribuição 4.0 Internaciona 


\section{Resumen}

Objetivos: Verificar y describir el conocimiento de alumnos de medicina sobre medicamentos inapropiados para ancianos conforme a los Criterios de Beers. Métodos: Estudio transversal con aplicación de cuestionarios a 102 alumnos matriculados del $9^{\circ}$ al $11^{\circ}$ semestre del curso de Medicina de la Universidad Luterana do Brasil - Canoas. Se levantó informaciones referentes al conocimiento de los académicos sobre medicamentos inapropiados para ancianos frágiles o con más de 80 años, basado en la clasificación del Criterio de Beers.

Resultados: $84,3 \%$ de los alumnos encuestados afirmaron tener conocimiento sobre medicamentos inapropiados para ancianos, pero el porcentaje total de aciertos fue del $20,2 \%$. El mayor número de aciertos fue relacionado con la seguridad de Diazepam (49\%) y el menor porcentaje de aciertos fue de la Fluoxetina, con apenas el 4,9\% de las respuestas correctas. El mayor porcentaje de respuestas "No Sé" fueron de Oxibutinina (48\%) y Ciclobenzaprina $(37,3 \%)$. Los entrevistados que afirmaron no conocer el tema y los individuos masculinos presentaron un conocimiento significativamente mayor $(p=0,001)$.

Conclusiones: La mayoría de los entrevistados afirman poseer conocimiento sobre el tema, pero la frecuencia de aciertos fue baja. El mejoramiento de la enseñanza, buscando mayor calidad en la prescripción y a del uso racional de medicamentos, es necesario para minimizar la iatrogenia en ancianos.

Palabras clave: geriatría, anciano, enfermedad latrogénica, utilización de medicamentos, tratamiento farmacológico, educación médica.

\section{Introdução}

A população idosa do Brasil vem em rápido crescimento devido ao declínio da taxa de natalidade e ao aumento da expectativa de vida no pais. A estimativa é de que o Brasil seja o sexto país em número de idosos em 2020, quando deve chegar a 30 milhões de pessoas com 60 anos ou mais, representando $13,6 \%$ da população geral ${ }^{1}$. O envelhecimento traz como consequência mais vulnerabilidade à saúde, desencadeando a presença de um número elevado de doenças agudas, crônicas e degenerativas, predispondo a pessoa idosa a um maior uso de medicamentos e, consequentemente, a um maior risco de iatrogenia?

A iatrogenia medicamentosa é considerada um dos temas conhecidos como sendo um dos "gigantes da geriatria", pois em idosos é mais frequente e, normalmente, de maior gravidade, em especial, no idoso frágil ou com mais de 80 anos. Isso porque esses pacientes apresentam alterações próprias do envelhecimento em relação à farmacodinâmica e à farmacocinética, que os tornam mais suscetiveis aos efeitos dos fármacos. A iatrogenia em geriatria se traduz nas reações adversas a medicamentos (RAMs), que representam um importante problema de saúde pública, estando associadas à morbimortalidade em pacientes idosos ${ }^{3}$. Nos Estados Unidos as RAMs estão presentes em 5 a 10\% das hospitalizações de pacientes idosos, sendo a quarta causa de morte 4 . No Brasil, um estudo em hospital público observou que $61,8 \%$ dos idosos internados apresentaram ao menos uma RAM ${ }^{3}$.

A polifarmácia representa o principal fator de risco para reações adversas a medicamentos e consequente iatrogenia em idosos, sendo essa relação ainda mais forte quando observada em pacientes idosos frágeis ou idosos longevos 5.6 .7 . Esse conceito se refere ao consumo de múltiplos medicamentos, muitas vezes, sem que esses apresentem clara correspondência entre a doença e a sua ação, e em esquemas terapêuticos duplicados. Idosos consomem em média de 2 a 5 fármacos, fato relacionado ao predomínio de doenças crônicas, à presença de múltiplas comorbidades e à existência de múltiplos prescritores observados na população idosa, onde a polifarmácia está presente em até 50\% 8.9 .

Esse uso irracional de medicamentos pode levar ao consumo de medicamentos potencialmente inapropriados para pacientes idosos (MIPIs), uma vez que os MIPIs são fármacos ou classes farmacológicas que apresentam mais risco de reações adversas a medicamentos em pacientes idosos? ${ }^{7}$. Estudos de vários paises apontam a polifarmácia como um fator de risco para a prescrição de medicamentos inapropriados e demonstram a alta prevalência do consumo de MIPIs por idosos ${ }^{10}$. No Brasil, um estudo demonstrou que $37,6 \%$ das 1.800 prescrições estudadas foram consideradas inapropriadas e que a probabilidade de prescrição de MIPIs aumenta em duas vezes se o consumo for de 5 a 6 medicamentos ${ }^{11}$. Uma pesquisa em seis hospitais universitários europeus demonstrou uma taxa global de 30,4\% de prevalência de MIPIs nas prescrições de pacientes idosos internados ${ }^{12}$.

Para definir os medicamentos que apresentam riscos à população idosa, a Sociedade Americana 
de Geriatria (AGS) desenvolveu uma listagem de medicações que promovem efeitos adversos em idosos, o Critérios de Beers. Essa ferramenta lista 53 medicamentos ou classes de medicamentos potencialmente inapropriados para idosos ${ }^{13}$. No Brasil foi comprovada a aplicabilidade do instrumento, Critérios de Beers, através de estudo que analisou a lista de medicamentos genéricos distribuidos no país e constatou que $6,7 \%$ do total de fármacos listados correspondiam aos medicamentos classificados pelo Critério como potencialmente inapropriado ${ }^{14}$.

Segundo o parecer da Organização Mundial da Saúde (OMS), a forma mais efetiva para melhorar a prescrição medicamentosa na atenção primária à saúde é a educação dos profissionais da área ${ }^{11}$. Tendo em vista o crescimento acelerado de idosos no Brasil e que a prescrição inapropriada para esses pacientes representa o dobro de risco de RAMs e risco de hospitalização, torna-se necessário que o tema seja abordado durante a graduação para que o médico tenha conduta adequada em relação aos pacientes idosos ${ }^{15,16,17}$. Esse estudo tem como objetivo levantar dados quanto ao conhecimento de alunos de graduação de um Curso de Medicina no estado do Rio Grande do Sul em relação aos medicamentos de uso rotineiro na prática médica, encontrados no Sistema Único de Saúde e que pertencem à listagem do Critério de Beers para uso em idosos.

\section{Métodos}

Estudo transversal contemporâneo sobre conhecimento dos alunos do curso de medicina utilizando um questionário autoaplicado precedido por um Termo de Consentimento Livre e Esclarecido. Os dados foram coletados entre os alunos do 9. ${ }^{\circ}$ ao $11 .^{\circ}$ semestre do Curso de Medicina da Universidade Luterana do Brasil - Canoas, no periodo de setembro de 2015. durante atividades acadêmicas em sala de aula. O questionário foi elaborado para a pesquisa com base na listagem de medicamentos inapropriados para pessoas idosas segundo os Critérios de Beers de 2012, usando medicamentos que constam na Relação Nacional de Medicamentos
(RENAME). O questionário é estruturado com perguntas sobre as características demográficas da amostra e sobre a segurança de uma lista de 21 medicamentos que são de uso comum em pacientes idosos e disponiveis no Sistema Único de Saúde (SUS). Para fins de minimizar possiveis respostas falsas, em cada questão foi mantida uma alternativa de resposta de desconhecimento sobre o medicamento. O aluno foi orientado a marcar apenas uma alternativa em cada questão, sendo elas: "seguro", "não seguro", "requer ajuste de dose" e "não sei". Estabelecemos como critério para decisão na resposta dos alunos o idoso longevo e o idoso frágil, independentemente das condições clínicas, mesmo que todas as medicações devam ser evitadas a pacientes maiores de 65 anos, considerando que o grau de recomendação é ainda mais forte para idosos longevos ou frágeis. Sendo idoso longevo, aquele com 80 anos ou mais e idoso frágil o que apresenta reserva funcional reduzida e maior vulnerabilidade aos eventos adversos por declínio de múltiplos sistemas fisiológicos ${ }^{8}$.

A partir dos dados levantados nos questionários, foi construido um banco de dados no programa Excel 2013, e para a análise dos dados utilizou-se o programa SPSS versão 21.0. Para as variáveis contínuas foram utilizados média e desvio padrão, para as categóricas, número de ocorrências e porcentagem. Para a correlação entre as variáveis foram utilizados os testes Qui-Quadrado para as frequências das variáveis categóricas e os testes $U$ de Mann-Whitney e de proporção de acertos para as variáveis com distribuição não normal, a partir dos testes de KolmogorovSmirnov e Shapiro-Wilk. Considerou-se o nivel de significância de 95\% ( $p<0,05)$.

O estudo foi submetido e aprovado pelo Comitê de Ética em Pesquisa da Universidade Luterana do Brasil, protocolo CAAE: 49105915.5.0000.5349.

\section{Resultados}

Dentre os 137 alunos matriculados durante o período do estudo, 102 responderam todo o questionário, sendo que 32 (31,4\%) foram respondidos por acadêmicos do $9 .^{\circ}$ semestre, 
$36(35.3 \%)$ do $10 .^{\circ}$ semestre e 34 (33.3\%) do $11^{\circ}{ }^{\circ}$ semestre, havendo uma harmonia percentual dos três semestres estudados. A idade média dos estudantes foi de 26,36 \pm 4,72 anos, tendo o indivíduo mais novo 21 anos e o mais velho 51 anos. Da amostra, 59,8\% eram do sexo feminino.

Em relação ao conhecimento prévio sobre medicamentos inapropriados para idosos, 84,3\% dos pesquisados afirmou ter tido informação ou contato com o tema, porém a porcentagem total de acertos foi de $20,2 \%$. A frequência e a distribuição percentual de respostas corretas para cada item da pergunta: "Qual o nivel de segurança da prescrição dos fármacos para um paciente idoso frágil ou de 80 anos ou mais" são mostradas na Tabela 1.

TABELA 1 - Frequência de respostas corretas para os itens da pergunta "Qual o nível de segurança da prescrição dos fármacos para um paciente idoso frágil ou idosos de 80 anos ou mais" dos indivíduos da amostra

\begin{tabular}{|c|c|c|c|c|}
\hline \multirow{2}{*}{$\frac{\text { Item }}{\text { Diazepam }}$} & \multirow{2}{*}{$\frac{\text { Resposta correta }}{\text { Não Seguro }}$} & \multicolumn{2}{|c|}{$\begin{array}{l}\text { Frequência de } \\
\text { acertos (\%) }\end{array}$} & \multirow{2}{*}{$\begin{array}{c}\begin{array}{c}\text { IC } 95 \% \text { para a } \\
\text { frequência de acertos }\end{array} \\
(39,4 ; 58,7)\end{array}$} \\
\hline & & 50 & (49) & \\
\hline $\begin{array}{l}\text { Reposição hormonal com } \\
\text { estrógeno oral }\end{array}$ & Não Seguro & 49 & (48) & $(38,5 ; 57,7)$ \\
\hline Digoxina & Requer Ajuste de Dose & 39 & $(38,2)$ & $(29,2 ; 47,9)$ \\
\hline Antidepressivos Tricíclicos & Não Seguro & 36 & $(35,3)$ & $(26,5 ; 44,9)$ \\
\hline Testosterona & Não Seguro & 34 & $(33,3)$ & $(24,7 ; 42,8)$ \\
\hline Laxantes de Contato & Não Seguro & 33 & $(32,4)$ & $(23,8 ; 41,8)$ \\
\hline Alprazolam & Requer Ajuste de Dose & 31 & $(30,4)$ & $(22,0 ; 39,7)$ \\
\hline Amiodarona & Não Seguro & 27 & $(26,5)$ & $(18,6 ; 35.5)$ \\
\hline $\begin{array}{c}\text { Aspirina como prevenção de } \\
\text { eventos cardiovasculares }\end{array}$ & Requer Ajuste de Dose & 25 & $(24,5)$ & $(16,9 ; 33,4)$ \\
\hline Nifedipino de longa duração & Seguro & 23 & $(22,5)$ & $(15,2 ; 31,3)$ \\
\hline $\begin{array}{l}\text { Anti-histaminicos de primeira } \\
\text { geração }\end{array}$ & Não Seguro & 21 & $(20,6)$ & $(13,5 ; 29,1)$ \\
\hline Metildopa & Não Seguro & 21 & $(20,6)$ & $(13,5 ; 29,1)$ \\
\hline Metoclopramida & Não Seguro & 21 & $(20,6)$ & $(13,5 ; 29,1)$ \\
\hline Clorpromazina & Não Seguro & 19 & $(18,6)$ & $(11,9 ; 26,9)$ \\
\hline Ciclobenzaprina & Não Seguro & 17 & $(16,7)$ & $(10,3 ; 24,7)$ \\
\hline Haloperidol & Não Seguro & 14 & $(13,7)$ & $(8,0 ; 21,3)$ \\
\hline Quetiapina & Não Seguro & 13 & $(12,7)$ & $(7,2 ; 20,1)$ \\
\hline Oxibutinina & Não Seguro & 12 & $(11,8)$ & $(6.5 ; 18.9)$ \\
\hline Glibenclamida & Não Seguro & 10 & $(9,8)$ & $(5,0 ; 16,6)$ \\
\hline Doxazosina & Não Seguro & 8 & $(7,8)$ & $(3.7 ; 14,1)$ \\
\hline Fluoxetina & Não Seguro & 5 & $(4,9)$ & $(1,8 ; 10,2)$ \\
\hline Resultado Geral & - & 508 & $(20,2)$ & \\
\hline
\end{tabular}


Em relação ao nível de segurança dos medicamentos ou classes medicamentosas, as opções que receberam maior percentual de respostas "Não Sei" foram as seguintes: Oxibutinina (48\%), Ciclobenzaprina (37,3\%), Testosterona (36,3\%) e Quetiapina (35.3\%). Os itens de medicamentos que embora não sejam seguros para pessoas idosas, porém receberam maior percentual de respostas "Seguro" foram: Fluoxetina (71,6\%), Aspirina (6o,8\%),
Doxazosina (53,9\%) e Glibenclamida (53,9\%). Os medicamentos mais marcados como "Não Seguros" foram: Diazepam (49\%), Reposição Hormonal com Estrógeno Oral (48\%), Antidepressivos Triciclicos (35.3\%) e Testosterona (33.3\%). Os mais marcados como "Requer Ajuste de Dose" foram: Haloperidol, Clorpromazina e Digoxina com 48\%, 37,3\% e 38,2\% de frequência, respectivamente, como expresso na Tabela 2.

TABELA 2 - Frequência de resposta para os itens da pergunta "Qual o nivel de segurança da prescrição dos fármacos para um paciente idoso frágil ou idosos de 80 anos ou mais" dos indivíduos da amostra

Frequência de Resposta Marcada

\begin{tabular}{|c|c|c|c|c|c|c|c|c|}
\hline \multirow{3}{*}{$\frac{\text { Item }}{\text { Diazepam }}$} & & & & & & & & \\
\hline & \multicolumn{2}{|c|}{ Seguro (\%) } & \multicolumn{2}{|c|}{ Não Seguro (\%) } & \multicolumn{2}{|c|}{$\begin{array}{c}\text { Requer Ajuste } \\
\text { na Dose }(\%)\end{array}$} & \multicolumn{2}{|c|}{ Não Sabe (\%) } \\
\hline & 15 & $(14,7)$ & 50 & (49) & 34 & $(33,3)$ & 3 & $(2,9)$ \\
\hline Antidepressivos Tricíclicos & 25 & $(24,5)$ & 36 & $(35,3)$ & 39 & $(38,2)$ & 2 & (2) \\
\hline $\begin{array}{l}\text { Anti-histaminicos de primeira } \\
\text { geração }\end{array}$ & 35 & $(34,3)$ & 21 & $(20,6)$ & 21 & $(20,6)$ & 25 & $(24,5)$ \\
\hline Testosterona & 15 & $(14,7)$ & 34 & $(33,3)$ & 16 & $(15,7)$ & 37 & $(36,3)$ \\
\hline Amiodarona & 26 & $(25,5)$ & 27 & $(26,5)$ & 33 & $(32,4)$ & 16 & $(15,7)$ \\
\hline Metildopa & 37 & $(36,3)$ & 21 & $(20,6)$ & 28 & $(27,5)$ & 16 & $(15,7)$ \\
\hline Nifedipino de longa duração & 23 & $(22,5)$ & 24 & $(23,5)$ & 32 & $(31,4)$ & 23 & $(22,5)$ \\
\hline Haloperidol & 24 & $(23,5)$ & 14 & $(13,7)$ & 49 & (48) & 15 & $(14,7)$ \\
\hline Clorpromazina & 21 & $(20,6)$ & 19 & $(18,6)$ & 38 & $(37,3)$ & 24 & $(23,5)$ \\
\hline Quetiapina & 24 & $(23.5)$ & 13 & $(12,7)$ & 29 & $(28,4)$ & 36 & $(35,3)$ \\
\hline Metoclopramida & 44 & $(43,1)$ & 21 & $(20,6)$ & 20 & $(19,6)$ & 17 & $(16,7)$ \\
\hline Laxantes de Contato & 32 & $(31,4)$ & 33 & $(32,4)$ & 16 & $(15,7)$ & 21 & $(20,6)$ \\
\hline $\begin{array}{l}\text { Reposição hormonal com } \\
\text { estrógeno oral }\end{array}$ & 19 & $(18,6)$ & 49 & (48) & 16 & $(15,7)$ & 18 & $(17,6)$ \\
\hline Ciclobenzaprina & 17 & $(16,7)$ & 17 & $(16,7)$ & 30 & $(29,4)$ & 38 & $(37,3)$ \\
\hline Fluoxetina & 73 & $(71,6)$ & 5 & $(4,9)$ & 21 & $(20,6)$ & 3 & $(2,9)$ \\
\hline Doxazosina & 55 & $(53,9)$ & 8 & $(7,8)$ & 15 & $(14,7)$ & 24 & $(23,5)$ \\
\hline Oxibutinina & 29 & $(28,4)$ & 12 & $(11,8)$ & 12 & $(11,8)$ & 49 & (48) \\
\hline Alprazolam & 30 & $(29,4)$ & 21 & $(20,6)$ & 31 & $(30,4)$ & 20 & $(19,6)$ \\
\hline Digoxina & 28 & $(27,5)$ & 24 & $(23,5)$ & 39 & $(38,2)$ & 11 & $(10,8)$ \\
\hline $\begin{array}{c}\text { Aspirina } \\
\text { (prevenção de evento } \\
\text { cardiovascular) }\end{array}$ & 62 & $(60,8)$ & 12 & $(11,8)$ & 25 & $(24,5)$ & 3 & $(2,9)$ \\
\hline Glibenclamida & 55 & $(53,9)$ & 10 & $(9,8)$ & 31 & $(30,4)$ & 6 & $(5,9)$ \\
\hline
\end{tabular}


O estudo observou que não teve relação estatística, considerando um nivel de confiança de 95\%, entre o semestre cursado e o número de acertos. Em relação ao número de acertos por sexo, o sexo masculino, apresentou conhecimento significativamente maior sobre o assunto, com média de rankings de 63,03 em comparação com o sexo feminino que apresentou 43,74, $\operatorname{com} \mathrm{p}=0,001$. Em relação ao conhecimento prévio sobre o tema, encontrou-se significância estatística em relação ao medicamento Oxibutinina, com p=0,001.

\section{Discussão}

No presente estudo os alunos apresentaram conhecimento pouco satisfatório em relação a segurança dos fármacos apresentados. $\mathrm{Na}$ prática médica também se observa a falta de conhecimento em relação à prescrição medicamentosa do paciente idoso. Vários estudos demonstram a ocorrência de prescrição inadequada para idosos em diferentes países e serviços de saúde. Um estudo de coorte americano observou as prescrições de pacientes idosos em 348 hospitais e constatou que $49 \%$ dos pacientes com mais de 65 anos receberam pelo menos um medicamento inapropriado, enquanto no Brasil 37,6\% das 1.800 prescrições estudadas foram consideradas inapropriadas ${ }^{18.19}$.

Segundo vários autores, as drogas inapropriadas mais prescritas para idosos são as de efeito no sistema nervoso central, principalmente o Diazepam, e os Antidepressivos Triciclicos. Nesse estudo o fármaco que apresentou o maior percentual de respostas corretas foi o Diazepam, $49 \%$ dos alunos afirmaram que o medicamento não é seguro para pacientes geriátricos, ainda assim essa frequência representa pouco menos da metade da amostra estudada. Esse benzodiazepínico de longa-ação causa sedação prolongada que aumenta o risco de quedas, além disso, seu uso prolongado está relacionado a efeitos adversos como angústia, dores articulares, depressão e tontura, resultando em prejuizos na funcionalidade do idoso ${ }^{19.20}$. Em relação aos antidepressivos triciclicos observamos uma frequência de acertos de apenas 35.3\%. Essa classe terapêutica causa efeitos sedativos associados à ocorrência de delirium e a quedas em idosos, além de efeitos cardíacos e de efeitos anticolinérgicos que podem agravar quadros comuns nesses pacientes, como incontinência urinária6,8.9.

Um dos fármacos mais prescritos em doses inapropriados para pacientes idosos é a digoxina, que em doses superiores a 0,125 miligramas é considerada de uso inapropriado pelo risco de intoxicação em pacientes idosos ${ }^{8}$. Estudos mostram que a toxicidade por digitálicos representa uma das principais causas de internações por efeitos adversos a medicamentos ${ }^{2}$. Nosso estudo demonstrou que somente 38,2\% dos alunos responderam corretamente que é necessário o ajuste da dose para a segurança na prescrição deste medicamento.

Chama atenção os medicamentos com menor número de acertos no estudo: Fluoxetina, Doxazosina e Glibenclamida. Estudos demonstram que estes medicamentos são comumente prescritos a idosos apesar de contraindicados ${ }^{9.21}$. A Fluoxetina apresentou somente 4.9\% de frequência de acertos. Apesar de ser uma opção reconhecida para o tratamento de doenças psiquiátricas em adultos e distribuída pelo SUS, esse fármaco apresenta questionável efetividade nas doses toleradas por pacientes idosos e deve ser evitado por apresentar meia-vida longa e potencial para interações medicamentosas, e decorrentes RAMs, por sua atuação no sistema enzimático do citocromo P450 8,20,22. A Doxazosina é considerada inapropriada com alto nivel de recomendação por apresentar elevado risco para hipotensão ortostática e por apresentar alternativas mais seguras ${ }^{13}$. O desempenho insatisfatório dos alunos quanto à segurança da Doxazosina - 7,8\% de acertos - pode ter relação com seu uso rotineiro em patologias da próstata devido o reconhecimento de sua eficácia em pacientes adultos ${ }^{8}$. O uso da Glibenclamida como tratamento da diabete está bem difundido e é considerada uma boa opção no que se refere a adultos jovens. Nesse estudo, 53,9\% da amostra classificaram essa sulfonilureia como segura para idosos, apesar de ela ser responsável pela reação 
adversa mais temida no tratamento do idoso com diabete, a hipoglicemia prolongada e grave $^{8}$.

O maior percentual de respostas "Não Sei" quanto ao nivel de segurança da prescrição foi observado nos medicamentos Oxibutinina com 48\%, Ciclobenzaprina com 37,3\%, Testosterona com 36,3\% e Quetiapina com 35,3\%. Essas respostas podem ter sido dadas frente ao desconhecimento dos alunos sobre a ação e a utilização desses fármacos. A Oxibutinina e a Ciclobenzaprina fazem parte de classes medicamentosas inapropriadas para idosos pela ação anticolinérgica e sedativa, aumentando o risco de queda em idosos ${ }^{13}$. O desconhecimento em relação à segurança da reposição hormonal com Testosterona pode ser devido à discussão ainda recente no meio médico sobre andropausa, e pouco abordada durante a graduação. Em relação à Quetiapina, neuroléptico de pouco efeito extrapiramidal, mas também considerado inapropriado, o desconhecimento por parte dos alunos pode estar relacionado à negligência ao estudo de doenças neurológicas, principalmente, patologias geriátricas como demência, levando a não familiarização ao fármaco e ao desconhecimento da aplicabilidade e dos efeitos dessa classe medicamentosa.

Outro aspecto interessante observado pelo estudo é a frequência de respostas quanto ao ajuste de dose das medicações. Dos entrevistados, 48\% marcaram a opção "Requer Ajuste de Dose" em relação ao Haloperidol. Este antipsicótico de primeira geração deve ser evitado para idosos por provocar sindromes extrapiramidais, aumento do risco de quedas e alteração na condução cardíaca. Entre os demais antipsicóticos, o Haloperidol é o mais contraindicado para a população idosa, sendo indicado apenas em quadros agudos e situações de emergência, fato que pode ter gerado o fator de confusão entre os entrevistados ${ }^{8,13}$.

Observou-se que os alunos que afirmaram não ter conhecimento prévio sobre medicamentos inapropriados para idosos foram os que apresentaram maior percentual relativo de acertos. Isto pode ser devido ao tamanho reduzido da amostra estudada ou ao fato de que o aluno subestima seu conhecimento por considerar o tema muito extenso, necessitando de maior discussão durante a graduação, ou não valoriza o tema a ponto de reconhecer como assunto já estudado.

Estudos que observaram o conhecimento de alunos sobre variados temas, como métodos diagnósticos de câncer de mama e morte encefálica, atribuíram ao baixo desempenho dos alunos entrevistados a reduzida importância dada para o assunto. Há temas ainda negligenciados durante a formação médica que necessitam de reparos quanto à metodologia de ensino e curricular ${ }^{23,24}$.

Estudos realizados no Canadá, Estados Unidos e Europa também observaram um déficit no ensino da farmacoterapia nas escolas médicas. A partir disso a Organização Mundial da Saúde (OMS) vem incentivando mudanças na metodologia do ensino, visando ao desenvolvimento de uma conduta prescritora adequada para cada paciente embasada por conhecimento científico fidedigno. Um estudo sobre o ensino do uso racional de medicamentos em uma escola médica brasileira, aponta que a mudança na metodologia em relação à formação da conduta prescritora é crucial para que os futuros profissionais tomem condutas através do raciocínio clínico e não apenas se baseado na experiência de seus preceptores ${ }^{25}$.

Quanto à significância estatística observada no número de acertos por sexo, não encontramos em nosso estudo justificativa para o fato de que o sexo masculino tenha apresentado maior número de acertos no questionário aplicado, com média de rankings de 63,03. O tamanho reduzido da amostra estudada pode ser uma razão para esse resultado. Uma melhor discussão sobre esse aspecto pode ser obtida através de outro estudo mais específico e excluindo-se outras variáveis.

\section{Conclusão}

Quanto ao conhecimento de estudantes de medicina dos últimos semestres da Universidade Luterana do Brasil - Canoas sobre medicamentos inapropriados para idosos, concluímos que os alunos apresentaram uma frequência baixa em percentual de acertos no questionário aplicado, apenas $20,2 \%$, apesar de $84,3 \%$ da amostra afirmarem ter conhecimento sobre o tema. 
Como possiveis explicações, relacionamos a falta de discussão sobre uso racional de medicamentos durante toda a formação médica e o aprendizado através da observação de condutas inadequadas para os pacientes idosos, porém ainda comuns, na prática médica. Não encontramos na literatura trabalhos semelhantes a esse, impossibilitando comparação similar. Portanto, mais estudos sobre educação médica em geriatria são necessários, além de discussões a respeito do conhecimento sobre prescrição adequada a idosos em todo o meio médico e acadêmico.

\section{Referências}

1. Cruz DT, Caetano VC, Leite IGC. Envelhecimento populacional e bases legais da atenção à saúde do idoso. Cad. Saúde Colet. 2010;18(4):500-8.

2. Neto PRO, Cuman RKN. Medicamentos potencialmente inapropriados para idosos e sua presença no SUS: Avaliação das Listas Padronizadas - Rev. Bras. Geriatr. Gerontol. 2011;14(2):285-29. https://doi. org/10.1590/S1809-98232011000200009.

3. Silva EA, Macedo LC. Polifarmácia em Idosos. Rev. S. Pesq. 2013:6(3):477-486.

4. Halter JB, Ouslander JG, Tinetti ME, et al. Hazzard's Geriatric Medicine and Gerontology. 5th ed. New York: McGraw-Hill; 2003

5. Paula TC, Bochner R, Montilla DER. Análise clínica e epidemiológica das internações hospitalares de idosos decorrentes de intoxicações e efeitos adversos de medicamentos, Brasil, de 2004 a 2008. Rev. Bras. Epidemiol. 2012;15(4):828-844. https://doi. org/10.1590/S1415-790X2012000400014.

6. Carvalho-Filho ET, Saporetti L, Souza MAR, Arantes ACLQ, Vaz MYKc, Hojaiji NHSL. et al. latrogenia em pacientes idosos hospitalizados. Rev. S. Púb. 1998;32(1):36-42. https://doi.org/10.1590/S003489101998000100005

7. Costa RM, Lima VAB, Paiva IG, Sousa PTP de, Lima LG. Uso de medicamentos por idosos: Algumas considerações. Rev. Bras. Geriatr. e Gerontol. 2008:3(2):126-13.

8. Cabrera M. Polifarmácia e Adequação do Uso de Medicamentos. In: Freitas EV, Py L., editores. Tratado de Geriatria e Gerontologia. $3^{a}$ ed. Rio de Janeiro. Guanabara Koogan; 2011. p 1055-1061.

9. Marin MJS, Cecílio LCO, Perez AEWUF, Santella F, Silva CBA, Gonçalves Filho R. et al. Caracterização do uso de medicamentos entre idosos de uma unidade do Programa de Saúde da Família. Cad.

10. Rozenfeld S. Prevalência, fatores associados e mau uso de medicamentos entre os idosos: uma revisão. Cad. Saúde Pub. 2003;19(3):717-24. https:// doi.org/10.1590/S0102-311X2003000300004.
11. Faustino CG, Martins MA, Jacob-Filho W. Medicamentos potencialmente inapropriados prescritos a pacientes idosos ambulatoriais de clínica médica Einstein. 2011; 9(1):18-23. https://doi.org/10.1590/ s1679-45082011a01844.

12. Gallagher P, Lang PO, Cherubini A, Topinková, E., Cruz-Jentoft, A., Montero Errasquín, B. et al. Prevalence of potentially inappropriate prescribing in an acutely ill population of older patients admitted to six European hospitals. Eur. J. Clin. Pharmacol. 2011;67(11):1175-88. https://doi.org/10.1007/s00228-011-1061-0.

13. American Geriatrics Society 2012 Beers Criteria Update Expert Panel. American Geriatrics Society updated Beers Criteria for potentially inappropriate medication use in older adults. J Am Geriatr Soc. 2012;6o(4):616-31. https://doi.org/10.1111/j. 1532-5415.2012.03923.x.

14. Gorzoni ML, Fabbri RMA, Pires SL. Critérios de Beers-Fick e Medicamentos Genéricos no Brasil. Rev Assoc Med Bras. 2008;54(4):353-6. https://doi. org/10.1590/S0104-42302008000400022.

15. Passarelli MC, Jacob-Filho W, Figueras A. Adverse drug reactions in an elderly hospitalised population: inappropriate prescription is a leading cause. Drugs Aging. 2005;22(9):767-77. https://doi. org/10.2165/00002512-200522090-00005.

16. Tavares FM. Reflexões acerca da iatrogenia e educação médica. Rev. Bras. Edu. Med. 2007:31(2):180-185. https://doi.org/10.1590/S010055022007000200010

17. Brasil. Ministério da Saúde. Uso Racional de Medicamentos Brasilia [internet]. Brasilia; 2012. [Capturado em 19 Mai 2014l. Disponivel em: http://bvsms.saude. gov.br/bvs/publicacoes/cartilha_promocao_uso_ racional_medicamentos.pdf

18. Rothberg MB, Pekow PS, Liu F, Korc-Grodzicki B, Brennan, MJ, Bellantonio, S. et al. Potentially inappropriate medication use in hospitalized elders. J Hosp Med. 2008;3(2):91-102. https://doi. org/10.1002/jhm.290.

19. Praxedes MFS, Filho PCPT, Pinheiro MLP. Identificação e análise de prescrições de medicamentos potencialmente inapropriados para idosos em uma instituição hospitalar. Cienc Cuid Saude. 2011:10(2):338-344. https://doi.org/10.4025/cienccuidsaude.v10i2.10214.

20. Bueno S, Bandeira VAC, Oliveira KR, COLET, CF. Perfil de uso de medicamentos por idosos assistidos pelo Programa de Atenção ao Idoso (P.A.I.) da UNIJUI. Rev. Bras. Geriatr. Gerontol. 2012;15(1):51-6. https://doi.org/10.1590/S1809-98232012000100006.

21. Carvalho MFC, Romano-Lieber NS, Bergsten-Mendes G, Secoli SR, Ribeiro E, Lebrão ML et al. Polifarmácia entre idosos do Municipio São Paulo Estudo SABE. Rev Bras Epidemiol. 2012;15(4):817-27. https://doi.org/10.1590/S1415-790X2012000400013.

22. Fick DM, Cooper JW, Wade WE, Waller JL, Maclean JR, Beers, MH. Updating the beers criteria for potentially inappropriate medication use in older adults. Arch Intern Med. 2003;163(22):2716-24. https://doi.org/10.1001/archinte.163.22.2716. 
23. Bitencourt AGV, Neves FBCS, Durães L, Nascimento DT, Neves NMBC, Torreão L de A et al. Avaliação do conhecimento de estudantes de medicina sobre morte encefálica. Ver. Bras. Ter. Intensiva. 2007:19(2):144-50. https://doi.org/10.1590/S0103$507 \times 2007000200002$.

24. Aires NM, Freitas-Júnior RD, Paulinelli RR, Freire, B Ln, Finotti MCCF., Terra, SM. Conhecimento dos métodos para diagnóstico do câncer de mama entre estudantes de medicina. Rev Bras Ginecol Obstet.1999:21(3):133. https://doi.org/10.1590/S010072031999000300003 .

25. Sousa IF, Biscaro A, Biscaro F, Fernandes, MS Uso Racional de Medicamentos: Relato de Experiência no Ensino Médico da Unesc, Criciúma/ SC.2009:34(3):438-45. https://doi.org/10.1590/ S0100-55022010000300014.

\section{Isadora Brandão da Silva}

Especialista pelo Programa de Residência Médica em Clínica Médica no Hospital Universitário da Universidade Luterana do Brasil (ULBRA, Canoas, RS, Brasil), médica pela ULBRA (Canoas, RS, Brasil).

\section{Geniffer Curtinaz Cardoso}

Especialista pelo Programa de Residência Médica em Medicina Interna no Hospital Geral de Caxias do Sul (UCS, Caxias do Sul, RS, Brasil), médica pelo Curso de Medicina da Universidade Luterana do Brasil (ULBRA, Canoas, RS, Brasil).

\section{Felipe Franco}

Acadêmico do Curso de Medicina da Universidade Luterana do Brasil (ULBRA, Canoas, RS, Brasil).

\section{Paulo Roberto Cardoso Consoni}

Mestre em Gerontologia Biomédica (PUCRS, Porto Alegre, RS, Brasil), professor adjunto do Curso de Medicina da Universidade Luterana do Brasil (ULBRA, Canoas, RS, Brasil).

\section{Endereço para correspondência}

Isadora Brandão da Silva

João Pedro Schimitt, 893

Bairro Rondônia, 93415-640

Novo Hamburgo, RS, Brasil 